\title{
Prevalencia de consumo de tabaco en estudiantes de bachillerato
}

\author{
Rodríguez-Ramírez JM*, Macías-Galaviz Ma Teresa*, Serna-Silva Carolina**, Méndez-Navarrete \\ Gabriela**, Gómez-Cardona Juan Pablo*, Rodríguez-Nava Víctor Federico*
}

\begin{tabular}{|c|c|}
\hline $\begin{array}{l}\text { Resumen } \\
\text { El consumo de tabaco es un factor de riesgo en las principales } \\
\text { causas de defunción en el mundo, especialmente enfermeda- } \\
\text { des isquémicas, cerebro vasculares, crónicas de vías respirato- } \\
\text { rias y tumores malignos. Las tendencias muestran aumento } \\
\text { de tasas de prevalencia de tabaquismo en adolescentes y } \\
\text { jóvenes. Objetivo: Determinar prevalencia del consumo de } \\
\text { tabaco en estudiantes de bachillerato. Material y Métodos: } \\
\text { Estudio descriptivo observacional transversal prospectivo. Se } \\
\text { aplicó la Escala Exploratoria de Consumo de Tabaco (EECT), } \\
\text { autoadministrado, que evalúa prevalencia de consumo de ta- } \\
\text { baco. Resultados: Prevalencia de consumo de tabaco de } 82 \% \text {, } \\
46.4 \text { puntos porcentuales arriba de la media nacional. Edad } \\
\text { de inicio del consumo de tabaco de I } 4.3 \text { años para fuma- } \\
\text { dores activos y exfumadores, } 2.8 \text { años más temprano que } \\
\text { los resultados de la ENA } 2008 \text {. La población estudiada se } \\
\text { distribuyó en } 3 \text { grupos: fumadores activos, exfumadores y no } \\
\text { fumadores representando } 59 \% \text {, } 23 \% \text { y I } 8 \% \text { respectivamente. } \\
\text { Conclusiones: El tabaquismo es un problema de salud públi- } \\
\text { ca con implicaciones graves a largo plazo en la salud de la } \\
\text { población; las tendencias muestran una relación inversamente } \\
\text { proporcional entre la edad de inicio en el consumo, la cual } \\
\text { disminuye presentándose cada vez a edades más tempra- } \\
\text { nas y la prevalencia de consumo, la cual evidencia un franco } \\
\text { incremento. Las acciones de salud deberán enfocarse a la } \\
\text { prevención primaria y principalmente a la población vulnera- } \\
\text { ble representada por niños, adolescentes y jóvenes. LuXMÉDICA, } \\
\text { AÑo } 7 \text { Núm } 21 \text { MaY0-AGosto } 2012 \text { pp 3-8 }\end{array}$ & $\begin{array}{l}\text { Abstract } \\
\text { Snuff consumption is a risk factor in the leading causes of } \\
\text { death worldwide, especially ischemic diseases, cerebrovascu- } \\
\text { lar, chronic respiratory and malignant tumors. Trends show } \\
\text { increasing prevalence rates of smoking among adolescents } \\
\text { and youth. Objective: To determine prevalence of snuff in } \\
\text { high school students. Material and Methods: Descriptive } \\
\text { Prospective Observational Cross. Scale was administered Ex- } \\
\text { ploratory Consumer Snuff (EECT), self-administered, which } \\
\text { assesses prevalence of snuff. Results: The prevalence of snuff } \\
\text { consumption of } 82 \% \text {, } 46.4 \text { percentage points above the na- } \\
\text { tional average. Age of first use of snuff from I } 4.3 \text { years for } \\
\text { smokers and former smokers, } 2.8 \text { years earlier than the re- } \\
\text { sults of the ENA } 2008 . \text { The study population was distributed } \\
\text { into } 3 \text { groups: smokers, former smokers and nonsmokers } \\
\text { representing } 59 \%, 23 \% \text { and I } 8 \% \text { respectively. Conclusions: } \\
\text { Smoking is a public health problem with serious long term } \\
\text { implications on the health of the population trends show an } \\
\text { inverse relationship between age at first use, which decreas- } \\
\text { es each time appearing at younger ages and prevalence of } \\
\text { use, which shows a frank increase. Health activities should } \\
\text { focus on primary prevention and especially to the vulnerable } \\
\text { population represented by children, adolescents and youth. } \\
\text { LUXMÉDICA, AÑ } 7 \text { NúM } 21 \text { MAY0-AGOSTO } 2012 \text { pp 3-8 }\end{array}$ \\
\hline & Keywords: consumption, smoking prevalence \\
\hline
\end{tabular}

* Profesores investigadores del Departamento de Enfermería del Centro de Ciencias de la Salud de la Universidad Autónoma de Aguascalientes ** Pasantes del servicio social de la licenciatura de enfermería

Fecha de recibido: 5 de marzo 2012

Fecha de aceptación: 29 de junio 2012

Correspondencia MCE José Manuel Rodríguez Ramírez, Departamento de Enfermería del Centro de Ciencias de la Salud de la Universidad Autónoma de Aguascalientes, Avenida Universidad 940, CP 20131. Aguascalientes, Ags., México. Teléfono 014499108437 correo electrónico victor-rguez7@hotmail.com 


\section{Introducción}

Las adicciones son uno de los principales problemas que se presentan en el campo de la salud pública, el tabaquismo se expresa por la necesidad compulsiva de consumir tabaco y la dificultad para abandonarlo; cuando existen estos elementos se puede determinar que existe una forma de dependencia al tabaco, ${ }^{1}$ distinguiéndose componentes físicos y psicológicos ${ }^{2}$ y representa un problema de difícil control por la aceptación social y de fácil acceso o disponibilidad.

El tabaquismo es una ventana para el inicio del consumo de alcohol o de otras drogas, siendo factor de riesgo en las principales causas de defunción en el mundo (enfermedades isquémicas, tumores malignos, enfermedad cerebrovascular, enfermedades crónicas de vías respiratorias) ${ }^{3}$.

De acuerdo con la Organización Mundial de Salud (OMS), en el siglo XX ocurrieron 100 millones de muertes atribuibles al tabaco y estima que, para el 2030 será un billón. La Organización Panamericana de la Salud (OPS), reporta que a largo plazo los fumadores tienen $50 \%$ de probabilidad de morir como consecuencia de una enfermedad relacionada con el consumo de tabaco, y de éstas casi la mitad ocurrirá entre la población de 35 a 69 años ${ }^{4}$; en total, la epidemia de tabaquismo mata cada año a casi 6 millones de personas, de proseguir esa tendencia, para 2030 la cifra aumentará hasta más de 8 millones anuales 5 .

En México, según los resultados de la Encuesta Nacional de Adicciones 2008 (ENA 2008), los estados que presentan la edad de inicio diario al consumo del tabaco más temprana, son San Luis Potosí y Jalisco (16.2 años), también revela que Aguascalientes es el estado con la mayor prevalencia de consumo de tabaco. En el estado, el $30.1 \%$ de la población de entre 12 y 65 años de edad es clasificada como fumador activo (191 mil personas) ${ }^{6}$. El Instituto Nacional de Estadística y Geografía (INEGI) informa que en México hay aproximadamente 31 millones de personas expuestas a los daños a la salud causados por el tabaco7.

Las últimas tendencias muestran un aumento de las tasas de prevalencia de tabaquismo entre niños, adolescentes y jóvenes, así como un comienzo precoz del hábito. El costo económico del tabaquismo es devastador, estudios revelan que en hogares pobres, los productos del tabaco representan hasta un $10 \%$ del gasto familiar, lo que implica menos dinero para cubrir necesidades básicas ${ }^{8}$. 


\section{Material y métodos}

Estudio descriptivo, observacional, transversal y prospectivo, realizado entre agosto 2010 a julio 2011 en una institución educativa privada, el Instituto Manuel Castelazo de Aguascalientes, Ags. No se realizó cálculo de muestra considerando el tamaño de la población, incluyendo así a la totalidad de los alumnos inscritos en el cuatrimestre mayo-agosto 2011 ( $n=98$ alumnos). Se les aplicó la "escala exploratoria de consumo de tabaco" (EECT), de forma autoadministrada, cuyo objetivo fue evaluar prevalencia de consumo de tabaco. La EECT constituye un estrato del utilizado en la Encuesta Nacional de Adic- ciones (ENA) 2008, incluyendo algunos indicadores de dependencia al tabaco de la Escala de Fagerström; realizado por la Secretaria de Salud (SSA), Consejo Nacional Contra las Adicciones (CONADIC), e Instituto Nacional de Psiquiatría "Ramón de la Fuente Muñiz" (INPRFM) (2003). Se solicitó autorización de las autoridades de la institución educativa, previo consentimiento informado y se aplicó la EECT. El análisis estadístico se realizó con el programa estadístico SPSS versión 19, se realizó estadística descriptiva para variables numéricas y nominales.

\section{| | | | | | | | | | | | | | | | | | | | | | | | | | | | | | | | | | | | | | | | | | | | | | | | | | | | | | | | | | | | | | | | | | | | | | | | | | | | | | | | | | | | | | | | | | | | | | | | | | | | | | | | | | | | | | | | | |}

\section{Resultados}

Se estudiaron 98 estudiantes, de los cuales $64.3 \%$ corresponden al sexo masculino y el $35.7 \%$ al sexo femenino; la prevalencia de consumo de alguna vez en la vida fue del $82 \%$; la edad promedio de inicio de consumo fue de 15 años en un $21.25 \%$ de los estudiantes. Los resultados mostraron que los fumadores activos representan el $59 \%$, los ex fumadores $23 \%$ y los no fumadores $18 \%$.De la población fumadora, el consumo de tabaco en el último año fue del $71 \%$, el consumo en el último mes alcanzó el $57.75 \%$ y por último el consumo en los últimos 5 días fue del $28.75 \%$. El $50 \%$ de los estudiantes refieren haber consumido más de 5 cajetillas durante su vida. El $46.25 \%$ de los estudiantes han consumido tabaco de manera ocasional y $26.2 \%$ diariamente. En relación con la cantidad de cigarros que consumen se encontró que $28.75 \%$ de los estudiantes fuma de 1 a 15 cigarros diariamente.
El $8.75 \%$ de la población fumadora consumen mayor cantidad de cigarrillos por las mañanas, $41.25 \%$ por las tardes o al anochecer; el $30 \%$ tarda más de 30 minutos después de levantarse en encender su primer cigarro y $5 \%$ menos de 30 minutos. El $58.75 \%$ de los alumnos no se abstiene de fumar en lugares prohibidos y únicamente el $7.5 \%$ se abstienen de fumar; el $85 \%$ no fuman cuando se enferman, el $15 \%$ de los alumnos sí consumen tabaco durante este período; el $58.75 \%$ de los alumnos han intentado dejar de consumir tabaco y $41.25 \%$ no han intentado dejar de fumar. El $96 \%$ de los estudiantes conviven con 1 a 5 personas fumadoras en casa, el $67 \%$ con 1 a 5 personas fumadoras en el trabajo y el $44 \%$ con de 1 a 5 personas fumadoras en el aula. Al 56\%, no les molesta que fumen cerca de ellos mientras que al $44 \%$ si les molesta que fumen cerca de ellos.(Tabla 1) 
Tabla I

Principales resultados asociados al estudio

\begin{tabular}{|c|c|c|}
\hline \multicolumn{2}{|c|}{ VARIABLES } & \multirow{2}{*}{$\begin{array}{c}\text { PORCENTAJE } \\
59 \\
\end{array}$} \\
\hline DISTRIBUCIÓN DE LA POBLACIÓN & Fumadores activos & \\
\hline ESTUDIADA SEGÚN CONSUMO DE & Ex-fumadores & 23 \\
\hline & No fumadores & 18 \\
\hline \multirow{3}{*}{$\begin{array}{l}\text { PREVALENCIA DE } \\
\text { CONSUMO } \\
\text { DE TABACO }\end{array}$} & Alguna vez en la vida & 82 \\
\hline & En el último año & 71 \\
\hline & En el último mes & 57.7 \\
\hline \multirow{2}{*}{$\begin{array}{l}\text { CONSUMO DE TABACO } \\
\text { DURANTE EL DÍA }\end{array}$} & Por la mañanas & 8.75 \\
\hline & Por las tardes o al anochecer & 41.25 \\
\hline \multirow{2}{*}{$\begin{array}{l}\text { TIEMPO DE CONSUMO DEL PRIMER } \\
\text { CIGARRILLO DESPUÉS DE LEVANTARSE }\end{array}$} & Más de 30 minutos & 30 \\
\hline & Menos de 30 minutos & 5 \\
\hline \multirow{2}{*}{$\begin{array}{l}\text { CONSUMO DE TABACO EN LUGARES } \\
\text { PROHIBIDOS }\end{array}$} & $\mathrm{Si}$ & 58.7 \\
\hline & No & 7.5 \\
\hline \multirow{2}{*}{$\begin{array}{l}\text { CONSUMO DE TABACO DURANTE } \\
\text { ENFERMEDAD }\end{array}$} & $\mathrm{Si}$ & 15 \\
\hline & No & 85 \\
\hline \multirow{2}{*}{$\begin{array}{l}\text { HA INTENTADO DEJAR } \\
\text { DE FUMAR }\end{array}$} & $\mathrm{Si}$ & 58.75 \\
\hline & No & 41.25 \\
\hline \multirow{3}{*}{$\begin{array}{l}\text { LUGAR DE CONVIVENCIA } \\
\text { CON } 1 \text { A } 5 \text { PESONAS } \\
\text { FUMADORAS }\end{array}$} & Casa & 96 \\
\hline & Trabajo & 67 \\
\hline & Aula & 44 \\
\hline \multirow{2}{*}{$\begin{array}{l}\text { MOLESTIA PORQUE FUMEN } \\
\text { CERCA DE ELLOS }\end{array}$} & $\mathrm{Si}$ & 44 \\
\hline & No & 56 \\
\hline
\end{tabular}

Fuente: Encuesta Exploratoria de Consumo de Tabaco.

Instituto Manuel Castelazo. 2011.

\section{Discusión}

De acuerdo con la ENA 2008:

- El $20.4 \%$ de la población son fumadores activos, $18.4 \%$ corresponde a exfumadores y $61.1 \%$ nunca han fumado tabaco, lo cual difiere con los resultados obtenidos en este trabajo, que muestran que el $59 \%$ son fumadores activos,
$23 \%$ son exfumadores y el $18 \%$ nunca han consumido tabaco.

- Los fumadores activos inician el consumo diario de cigarro a los 16.7 años, los hombres a los 16 años y las mujeres a los 18 años de edad, lo cual difiere con los resultados obtenidos, ya que los hombres inician el consumo a los 14.2 
años y las mujeres a los 14.5 años, por lo que el inicio de consumo diario se presenta a edades más tempranas en la población estudiada, comparada con los datos de la ENA 2008

- El $20.4 \%$ de la población adulta ha fumado más de 5 cajetillas en su vida, mientras que el $50 \%$ de la población estudiada ha fumado la misma cantidad de cigarros, $29.6 \%$ más que la ENA 2008

- La edad promedio de consumo de tabaco por primera vez fue a los 13.7 años, difiriendo con la edad de inicio del consumo de la población estudiada donde la edad promedio fue de 14.3 años.

- El 85.3\% de los fumadores activos consumen menos de 16 cigarros diarios, $11.7 \%$ consumen de 16 a 25 y cerca del $3 \%$ consumen más de 25 cigarros por día; mientras que los datos obtenidos en el estudio el $28.75 \%$ de la población fumadora consumen menos de 16 cigarros diarios, el $1.25 \%$ de 16 a 25 y $1.25 \%$ fuman más de 25 cigarros por día.

De acuerdo con el INEGI, el principal lugar de exposición al humo de tabaco es el hogar, coincidiendo con los resultados encontrados por nosotros, pues del total de la población estudiada, 96\% conviven con 1 a 5 personas fumadoras en casa, $67 \% 1$ a 5 personas fumadoras en el trabajo y $44 \%$ con 1 a 5 personas fumadoras en el salón de clases.
A pesar de que existen normativas dirigidas a proteger a los no fumadores y prevenir el uso del tabaco emitidas por diversos organismos, como la OMS, para reducir en el número de muertes proyectadas para los próximos 20 años ${ }^{9}$, la estrategia MPOWER de la OMS para elevar los impuestos al tabaco ${ }^{10}$, la Ley General para el control del tabaco emitida por la Cámara de Diputados del H. Congreso de la Unión para proteger la salud de la población del efecto nocivo del tabaco ${ }^{11}$ y el Reglamento sobre Consumo de Tabaco para proteger la salud de las personas de los efectos nocivos del tabaco en lugares públicos cerrados ${ }^{12}$, nosotros encontramos que del total de la población estudiada, destaca que el $58.75 \%$ de los alumnos no se abstiene de fumar en lugares prohibidos y que el $15 \%$ de los alumnos consumen tabaco aún cuando se enferman. Lo anterior sugiere que abstenerse de fumar no depende de la normatividad sino de la permisividad y aprendizaje por observación (modelamiento) en casa.

En el estudio "Prevalencia y factores asociados al consumo de tabaco en adolescentes de una preparatoria de Guadalajara, Jalisco, México" Nuño-Gutiérrez et al ${ }^{13}$ encontraron que la prevalencia de consumo de tabaco alguna vez en la vida fue de $58.2 \%$, en el último año $32.4 \%$ y en el último mes $23.9 \%$, los resultados del presente estudio se encuentran 23.8, 38.6 y 33.8 puntos porcentuales respectivamente por encima de los obtenidos en el estudio en referencia.

\section{Conclusiones}

El tabaquismo es un problema de salud pública con implicaciones graves a largo plazo en la salud de la población; las tendencias muestran una relación inversamente proporcional entre la edad de inicio en el consumo, la cual disminuye presentándose cada vez a edades más tempranas y la prevalencia de consumo, la cual evidencia un franco incremento. Las acciones de salud deberán enfocarse a la prevención primaria y principalmente a la población vulnerable representada por niños, adolescentes y jóvenes. 


\section{Bibliografía}

1. México. Instituto Nacional de geografía y estadística. Estadísticas sobre tabaquismo. Disponible en: [http://www.inegi.gob.mx/inegi/contenidos/espanol/prensa/contenidos/estadisticas/2007/tabaco07. pdf. Consultado el 25 de octubre de 2010

2. Organización Mundial de la Salud. Temas de salud tabaquismo. Disponible en: http://www.who.int/topics/tobacco/es/. Consultado el: 17 de octubre de 2010

3 Iglesias, Elisardo Becoña.Bases Teóricas que sustentan los Programas de Prevención de Drogas. págs. 40-41.

4. Organización Mundial de la Salud. Riesgo para la salud de los jóvenes. Disponible en: http://www.who. int/mediacentre/factsheets/fs345/es/index.html. Consultado el 15 de octubre de 2010

5. Organización Mundial de la Salud. La epidemia de tabaquismo mata cada año a casi 6 millones de personas. Disponible en: http://www.who.int/features/factfiles/tobacco_epidemic/tobacco_epidemic facts/es/index.html. Consultado el 17 de octubre de 2010

6. México. Comisión Nacional contra las Adicciones. Encuesta Nacional de Adicciones 2008. Disponible en: [http://www.conadic.salud.gob.mx/pdfs/ ena08/ENA08_NACIONAL.pdf] Consultado el 6 de Octubre 2010

7. México. Instituto Nacional de Geografía y Estadística. Estadísticas a propósito del día mundial sin tabaco. Disponible en: http://www.inegi.org. $m x /$ inegi $/$ default.aspx?s=inegi $\& c=2760 \& e=03 \&$ pred $=1$. Consultado el 25 de octubre de 2010
8. Organización Mundial de la Salud. Costes económicos del tabaco. Disponible en: http://www.who.int/ features/factfiles/tobacco_epidemic/tobacco_epidemic_facts/es/index9.html. Consultado el: 15 de octubre de 2010

9. Organización Mundial de la Salud.10 Datos sobre la epidemia de tabaquismo y el control mundial del tabaco. Disponible en: http://www.who.int/features/ factfiles/tobacco epidemic/es/index.html. Consultado el: 15 de octubre de 2010.]

10. Organización Mundial de la Salud. Iniciativa liberarse del tabaco. Disponible en: http://www.who.int/tobacco/mpower/package/es/index.html. Consultado el de noviembre de 2010

11. México. Secretaria de Salud del Estado de San Luis Potosí. En qué consiste la Ley general para el control de tabaco. Disponible en: http://www.slpsalud.gob. $\mathrm{mx} /$ coepris/fomento-sanitario/429-en-que-consiste-la-ley-general-para-el-control-del-tabaco.html. Consultado el 6 de octubre de 2010

12. México. Secretaria de Salud. Reglamento sobre consumo de tabaco. Disponible en: http://www.salud gob.mx/unidades/cdi/nom/compi/rsct.html. Consultado el 25 de Noviembre de 2010

13. Nuño-Gutiérrez BL, Alvarez-Nemegyei J, Madrigal De León $\mathrm{E}$, Rasmussen-Cruz B, "Prevalencia y factores asociados al consumo de tabaco en adolescentes de una preparatoria de Guadalajara, Jalisco, México". Salud Mental, 2005; 28(005):64-70. 